\title{
EL PAPEL DE LA GRAN PROPIEDAD MILITAR EN EL PROCESO DE CONSTRUCCIÓN DE LA CIUDAD ITALIANA. El caso particular del barrio Flaminio-Guido Reni.
}

\author{
Federico Camerin ${ }^{1}$ \\ Instituto Universitario de Urbanística de la Escuela Técnica Superior de Arquitectura, UVA Valladolid \\ (España) \\ Director de la tesis en curso: Catedrático y Profesor Emérito Alfonso Álvarez Mora \\ federico.camerin@uva.es
}

\begin{abstract}
RESUMEN
La investigación aborda el análisis del proceso de construcción de la ciudad italiana durante el del último tercio del siglo $\mathrm{XX}$ y principios de $\mathrm{XXI}$, a partir de las grandes propiedades procedentes de las instalaciones militares desmanteladas

El trabajo analiza la gran propiedad militar en el contexto urbano, su condición de "capital fijo en suelo", del que se requieren nuevas rentabilidades, tanto económicas como sociales. Es, en este sentido, cómo se prestará especial atención a la relación entre "gran propiedad militar", "agentes inmobiliarios" y "capital financiero". Parece evidente que, a partir del fenómeno de abandono que protagonizan, se produce una relación estrecha entre propiedad, financiación y promotores inmobiliarios. La propiedad, que antes tenía una destinación de uso, deja de tenerlo cuando este uso entra en posesión de un promotor inmobiliario, de una entidad financiera, del especulador habitual, en una palabra, del "gran capital", que se encarga de la realización de nuevas actuaciones urbanas.
\end{abstract}

Palabras clave: gran propiedad militar, materialización-producción, desmantelamiento-vaciamiento, regeneración urbana

\begin{abstract}
The research deals with the analysis of the Italian city construction process of the three last decades of the 20th century and the beginning of the 21st century starting from the great properties coming from dismantled military installations.

This work analyzes military great property in urban context, its condition of "soil capital", from which new economic and social returns are required. It is, in this sense, how special attention will be paid to the relationship between "great military property", "real estate agents" and "financial capital". It seems evident that, based on the military abandonment phenomenon, it exists a close relationship between property, financing and real estate developers. The property, with a previous use, when it ceases, comes into the possession of a real estate developer, a financial institution, the usual speculator, in a word, the "great capital", is responsible for the realization of new urban actions
\end{abstract}

Key words: military great property, materialization-production, dismantling-emptying, urban regeneration

\footnotetext{
${ }^{1}$ This work has been realized within the European Joint Doctorate “urbanHIST”. This project has received funding from the European Union's Horizon 2020 research and innovation programme under the Marie Skłodowska-Curie grant agreement No 721933.
} 


\section{Introducción}

A partir de la Unificación del Estado italiano, en 1861, sus ciudades se han caracterizado, históricamente, entre otras cuestiones, por la presencia de instalaciones militares. Realizadas en el marco de la nueva organización de la administración del Estado, estas grandes propiedades han marcado, profundamente, el contexto urbano donde se ubican, marcando la estructura de sus barrios, así como condicionando la dinámica comercial y del mercado inmobiliario. Procedentes de edificios religiosos, o de herencias nobiliarias preexistentes, incluso, de nueva construcción, desde la segunda mitad del siglo XIX las propiedades militares se han configurado como propiedades demandadas por actividades y funciones de defensa nacional que, para hacer real su materialización espacial, necesitaban del consumo de una gran cantidad de suelo, marcando sus diferencias con respecto a aquellas otras propiedades designadas para otros tipos de uso (industrial, comercial, residencial, equipamientos, etc.).

Después de un largo período caracterizado por la estabilidad de uso de esas grandes propiedades, a finales de 1980 se ha producido una ruptura en los países europeos. En el marco del proceso de reestructuración de los sistemas de defensa, en relación con los cambios en el equilibrio geopolítico internacional desde el final de la Guerra Fría (Markusen y Judken, 1992), de un cambio en la gestión del patrimonio inmobiliario público hacia la privatización (Vaciago, 2007; Christophers, 2016; Whiteside, 2017) y de las nuevas políticas urbanas neoliberales de regeneración urbana (Harvey, 2005), muchas instalaciones militares se han ido abandonando para ser destinadas a otros usos (Servizio Studi Senato, 2007: 27-36). En el caso particular italiano, se han utilizado la enajenación y la puesta a la venta en el mercado inmobiliario de estos bienes para hacer frente, principalmente, a la Deuda pública, sin una conexión con las políticas de "regeneración urbana", ni con aquellas otras vinculadas a la producción del conocimiento, ni con las relacionadas con el territorio al que pertenecen las instalaciones militares. De hecho, en la década de 1990 y 2000 se han puesto de manifesto diferentes problemas a propósito de la reconversión del patrimonio militar, en general, público (Gastaldi, Camerin, 2012), que hoy día parecen estar superados a través de nuevos procedimientos y acuerdos entre las administraciones públicas, a nivel estatal (el Ministerio de Defensa y la Agencia del Dominio Público italiana) y local (los Ayuntamientos), con el soporte de promotores inmobiliarios, personificados con los fondos inmobiliarios (como el F.I.V., Fondo Investimenti per la Valorizzazione-Comparto Extra, de la sociedad de gestión de activos "Cassa Depositi e Prestiti sgr").

Con la finalidad de abordar el proceso de construcción de la ciudad italiana durante el último tercio del siglo XX y principios del siglo XXI, a partir de la gran propiedad militar, establecemos, en cuanto a la metodología, tres momentos referidos a otros tantos procesos que han afectado a las ciudades:

a) Primer momento. Para entender la ciudad de hoy hay que volver atrás y analizar la materializaciónproducción de los grandes artefactos militares que necesitan de la gran propiedad para espacializarse. Se explica cómo se materializan, cómo se producen, estos grandes artefactos que necesitan de una gran propiedad, es decir, se explica la construcción del objeto que demanda una gran propiedad para materializar su función;

b) Segundo momento. El desmantelamiento-vaciamiento en el marco del proceso de desposesiónapropiación de la gran propiedad que representa. Se explica, y se analiza, críticamente cómo se elabora una estrategia que va a procurar el vaciamiento, o la obsolescencia, de las instalaciones militares que va a desembocar en un proceso de desposesión-apropiación por parte de otros agentes inmobiliarios (el Fondo FIV-Comparto Extra) que no tienen nada a que ver con los primitivos, en este caso, el Ministerio de Defensa);

c) Tercer momento. La reinterpretación, reconstrucción-regeneración, de las grandes propiedades militares desmanteladas, a través de "proyectos urbanos", en el marco de procesos concretos de regeneración urbana. Vamos a referirnos al caso del cuartel Guido Reni, en el barrio Flaminio de Roma, instalación militar en estado de abandono que, en 2013, ha sido cedida a un fondo de inversión inmobiliario para la realización de la "Ciudad de la Ciencia", en el marco del más amplio proyecto urbano de 2005 Ilamado "El Barrio de la Ciudad de la Ciencia-el Proyecto Urbano Flaminio".

\section{2) La materialización-producción de las instalaciones militares en Italia}

La construcción de las instalaciones militares se ha relacionado, inevitablemente, con las dinámicas de desarrollo urbano. A finales del siglo XIX, con la industrialización, las ciudades europeas y, entre ellas, las italianas, bordeaban las antiguas murallas, las cuales sancionaban el límite entre las áreas urbanizadas y las rurales. En esta época, las ciudades comienzan a conocer importantes fases de expansión, estructurándose alrededor de algunos elementos urbanos clave. La realización de una red de 
carreteras y ferrocarriles, bien estructurada en el primer período posterior a la creación del Estado italiano (sobre todo, en el norte del país, más industrializado), convirtió a muchas de las ciudades italianas en lugares aptos para ubicar nuevos asentamientos militares, confiriendo al territorio nacional una estructura organizativa unitaria, también, desde el punto de vista militar, debido a la difusión del servicio militar obligatorio (Melis, 1941). La implantación de las instalaciones militares en la inmediata periferia de la ciudad, donde siempre se podía disponerse de suelo barato, dio lugar, a su vez, a nuevas transformaciones urbanísticas, lo que supuso la urbanización del contexto espacial donde se ubicaban dichas instalaciones.

En cuanto a la ubicación de los acuartelamientos, las nuevas condiciones sociales, económicas y políticas del nuevo Estado requerían, por un lado, asumir situaciones precedentes con respecto a los activos militares de los estados anteriores a la unificación y, por otro, cumplir los objetivos de un nuevo sistema de defensa nacional. Todo eso, con el objetivo de distribuir las Fuerzas Armadas en el territorio con un diseño orgánico que facilitase la supervisión de las plazas más importantes, la protección de las fronteras y las rutas de acceso al territorio nacional, el movimiento y la convergencia de tropas en caso de guerra (Bettinaria, 1989).

Por lo que respecta a los asentamientos militares, se establecen criterios con respecto al alejamiento de los barrios obreros pobres y sucios (habitados por los trabajadores, considerados verdaderos "centros de infección"); razones higiénicas y sanitarias (por disponer de aire más puro); libertad para gozar de una mayor amplitud (y desarrollar los edificios y los espacios abiertos) y razones económicas (por el menor precios de los terrenos), criterios que han contribuido a localizar los cuarteles fuera de los centros contaminados de la ciudad, alejados de los últimos suburbios, cerca de los campos, pero sin distanciarse demasiado de los centros históricos para evitar el aislamiento de la vida urbana al soldado, lo que repercutiría en su ánimo (Bruni, 1937).

Generalmente, en el período entre finales del siglo XIX y en los veinte años fascistas, los cuarteles han sido ubicados en el perímetro de las ciudades históricas, fuera del recinto amurallado ya demolido en casi todas sus partes, a lo largo de las vías de comunicaciones urbanas y extraurbanas, cerca de las vías ferrocarriles principales. Por razones estratégicas y de costes, en las ciudades capitales y en las plazas fortificadas, a menudo se han construido distritos militares enteros, como en Turín, en el barrio de Santa Rita, alrededor de la Plaza de las Fuerzas Armadas, en Roma, a lo largo de la Avenida de las Milicias y la zona de Cecchignola, en Milán en vía Monti y vía de las Fuerzas Armadas (por una clasificación de las tipologías de acuartelamiento realizadas entre 1861 y la segunda guerra mundial, véanse Turri, Cappelletti y Zamperini, 2008: 73). 


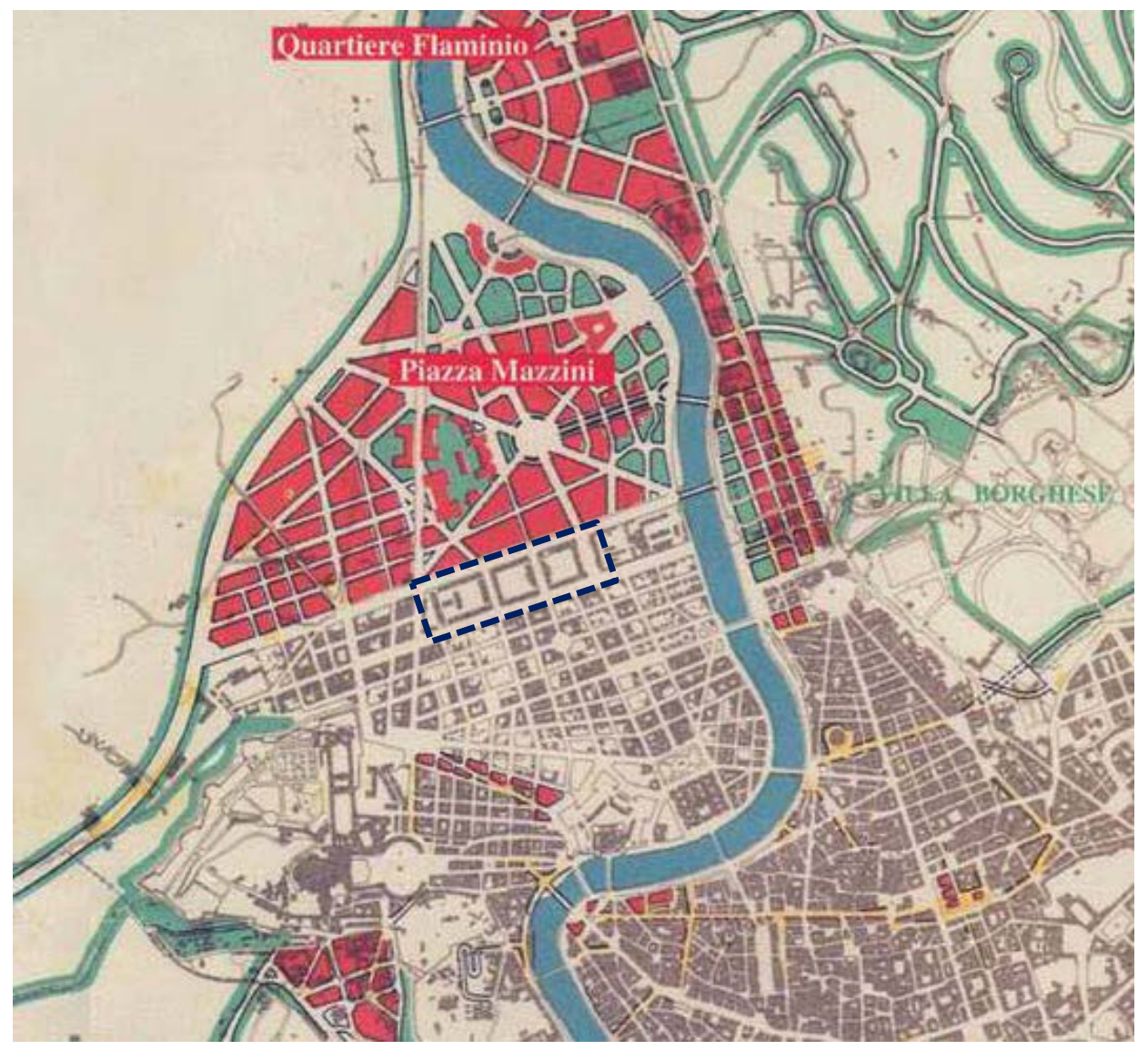

Extracto del Plan Regulador General de Roma de 1909. En azul el proyecto de acuartelamiento de la Avenida de las Milicias al sur de Piazza Mazzini.

Elaboración propia a partir del Plan Regulador General de Roma de 1909

Con el estallido de la Segunda Guerra Mundial, la localización planteada en las precedentes décadas ha demostrado su enorme vulnerabilidad a los ataques aéreos y se ha puesto en crisis. Por lo tanto, se han preferido soluciones fuera de la ciudad para reducir el reconocimiento de los complejos militares desde el alto y permitir una mayor libertad para maniobras y ejercicios. Sucesivamente, en la segunda posguerra, el peligro de un ataque atómico ha contribuido a repensar radicalmente la distribución de las instalaciones militares en el territorio italiano, de modo que las nuevas directrices han proporcionado espacios para unidades operativas más pequeñas que no excedían a un regimiento o batallón de las diferentes armas y cuerpos, distancias mínimas de 3 a $5 \mathrm{~km}$ entre dos instalaciones y racionalización de los asentamientos militares en una sola ciudad (Turri, Cappelletti y Zamperini, 2008: 76). Estas pautas sólo se han aplicado en parte, ya que no era posible completar la renovación de las propiedades inmobiliarias militares existentes. Además, frente a los acontecimientos de la segunda posguerra, el Tratado del Atlántico Norte, (que constituye la North Atlantic Teatry Organization, la NATO) del 4 de abril de 1949, el rearme alemán, el Pacto de Varsovia del 14 mayo de 1955 y la independencia y neutralización de Austria, a partir de mayo de 1945, se ha abierto una nueva fase para las Fuerzas Armadas italianas (Jean, 1989). Esto ha condicionado determinadas reorganizaciones y reestructuraciones del Ejército de Tierra, de la Armada y del Ejército del Aire, entre 1948 y 1954, en 1968 y en 1975 (Malatesta, Trevisan, Pozza y De Castro, 2015: 32-48), así como un consistente aumento de los gastos en el sector militar (por ejemplo, para la dotación de misiles Nike al Ejército del Aire en las décadas de 1950 y 1960 y los misiles Hawk al Ejércitos de Tierra en la década de 1970), el fomento de la industria militar y la comercialización de armas. Todo eso ha implicado una extensión de la presencia militar en el territorio italiano que ha incluido el establecimiento de las Fuerzas Armadas Americanas, por ejemplo, con las instalaciones de los comandos americanos de las fuerzas armadas de tierra Headquarters of the Allied Land Forces Southern Europe (HQ - LANDSOUTH) en Verona en 1951 y aquella con misiles nucleares Southern European Task Force (SETAF) en Vicenza, respectivamente en 1951 y 1955. 

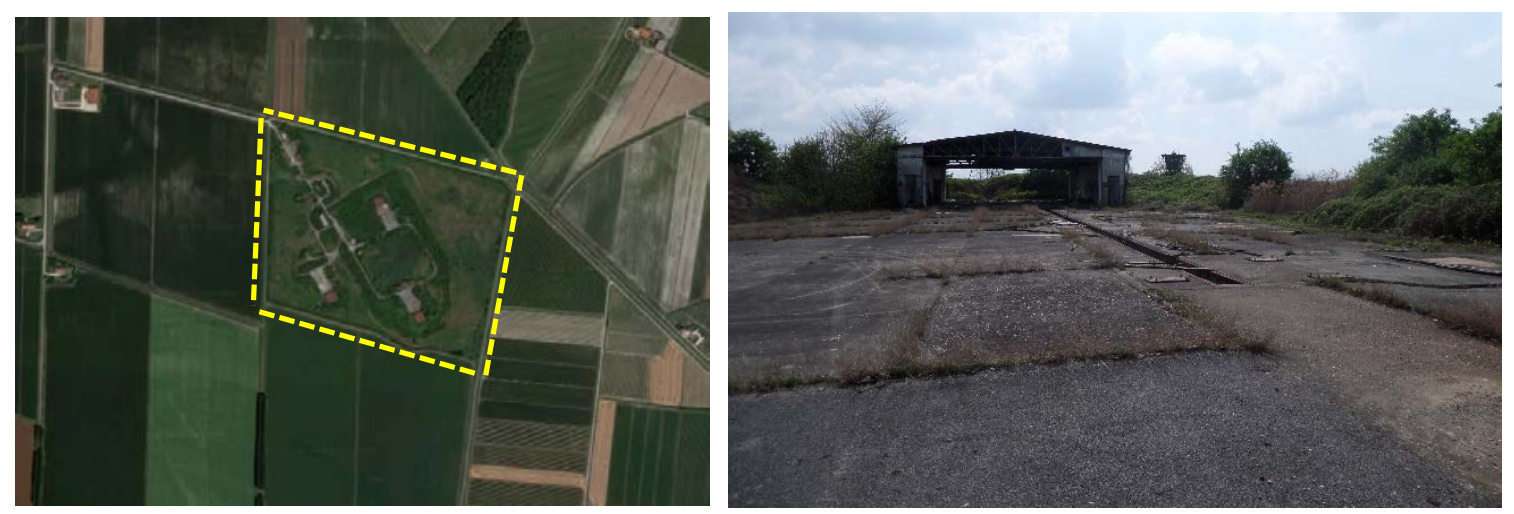

Ejemplo de área militar materializada en áreas periféricas, después de la Segunda Guerra mundial. A la izquierda, imagen de satélite y, a la derecha, fotografía del área interna de la antigua base militar "Zona de lanzamiento $57^{\circ}$ Gr. I.T." con misiles Nike de Ceggia (provincia de Venecia).

Fuente: Google Maps, 2018 y fotografía de Federico Camerin, 2017.

Enfocando la atención en el ámbito urbano, durante el largo periodo de materialización de las instalaciones militares en las ciudades italianas, las grandes propiedades se han mostrado, principalmente, de tres maneras:

1) reutilizando propiedades de origen monástico ubicadas dentro del núcleo histórico consolidado. Se trata de propiedades de gran dimensión, de interés histórico, arquitectónico y cultural, generalmente sujetos a vínculos de protección históricos y culturales, que se han reconvertido en instalaciones militares durante la segunda mitad del siglo XIX. De esta manera, los antiguos edificios, procedentes de uso religioso, no se han visto afectados por demoliciones, transformaciones y degradación.
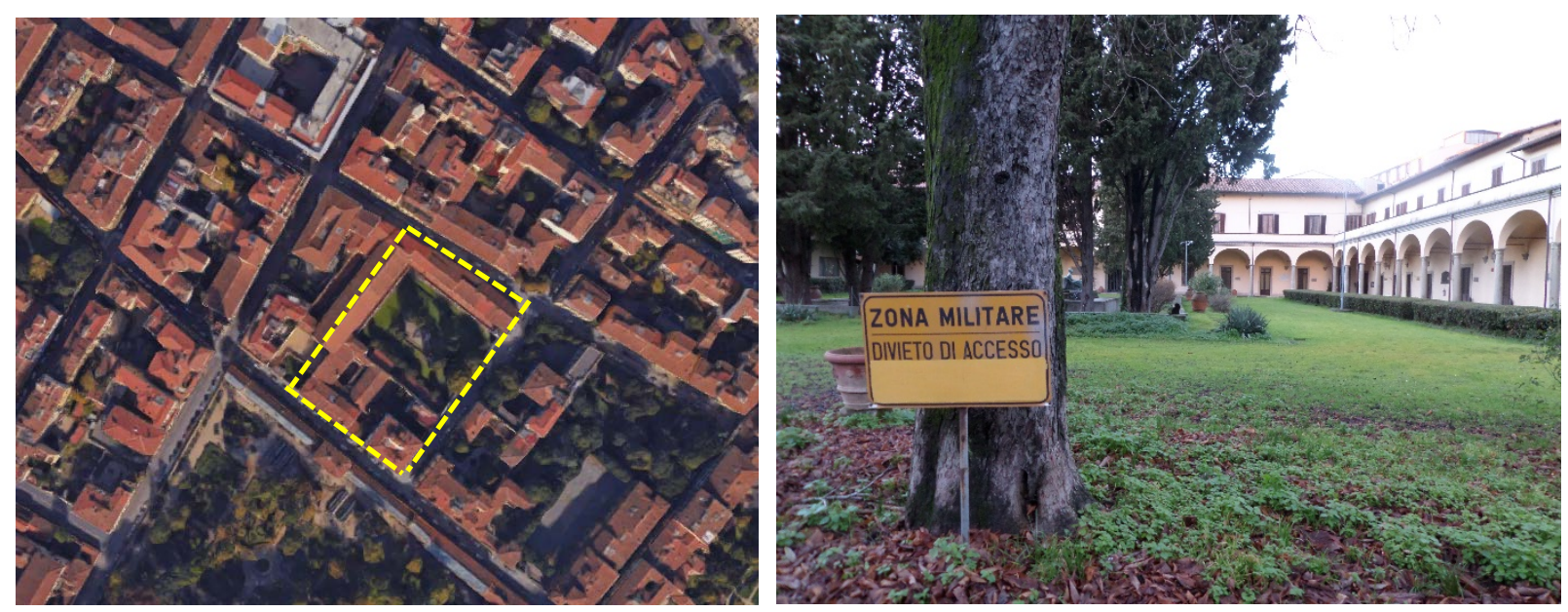

A la izquierda, imagen de satélite y, a la derecha, fotografía del cuartel Redi (Florencia), antiguo monasterio construido en 1297.

Google Maps, 2018 y fotografía de Federico Camerin, 2017.

2) Propiedades ubicadas en el borde del núcleo urbano de antigua formación, generalmente, alrededor de la circunvalación interior. Estas áreas se han visto, paulatinamente, afectadas por el proceso de urbanización, encontrándose, hoy día, reabsorbidas en el tejido urbano. 

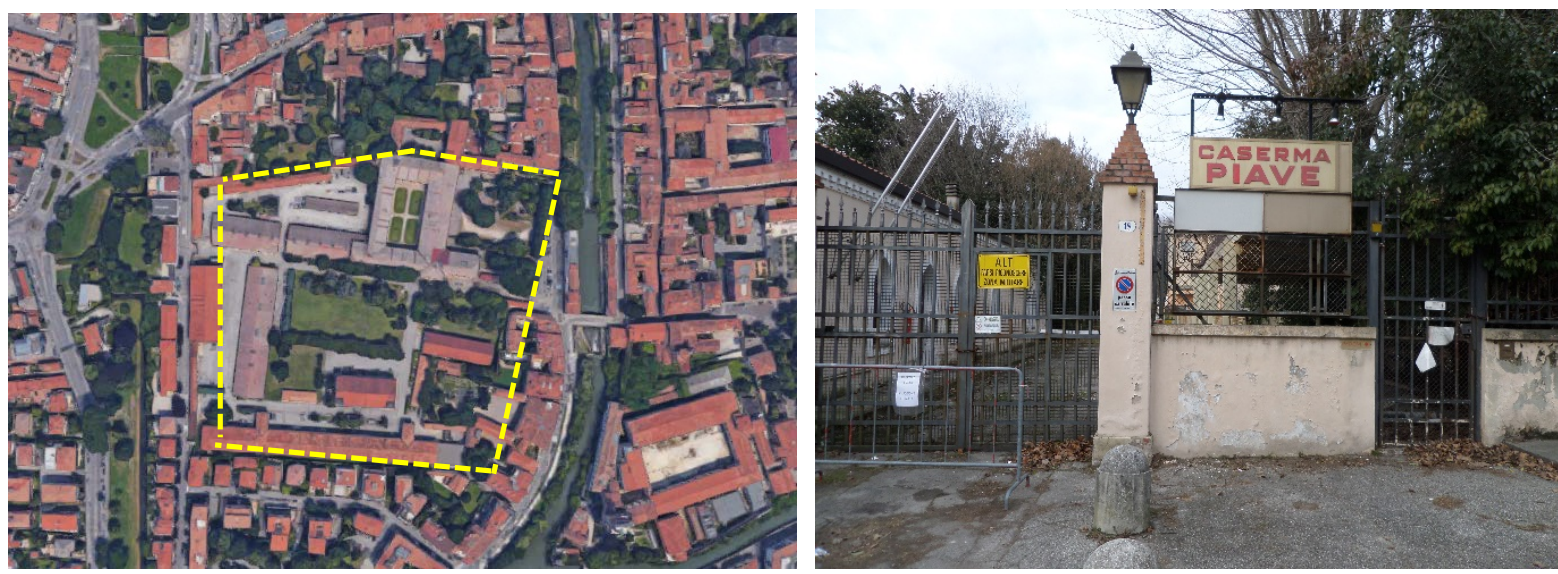

A la izquierda, imagen de satélite y, a la derecha, fotografía del cuartel Piave (Padua), realizado en la segunda mitad del siglo XIX dentro de la antigua circunvalación de la ciudad.

Google Maps, 2018 y fotografía de Federico Camerin, 2017.

3) Asentamientos militares construidos en zonas que, en el momento de su realización, se localizaban fuera del centro habitado, dentro de un radio de un kilómetro. Los cuarteles realizados en época fascista son los que se caracterizan por una mayor distancia con respecto a los núcleos históricos: eso se debió, también, al crecimiento de los centros de las ciudades durante el primer período posterior a la Unificación. Actualmente, estos bienes están absorbidos en áreas semi-periféricas o periféricas de las ciudades, predominantemente residenciales o, a veces, en ámbitos productivos industriales y artesanales, definidos por tejidos edilicios no consolidados y fragmentados.
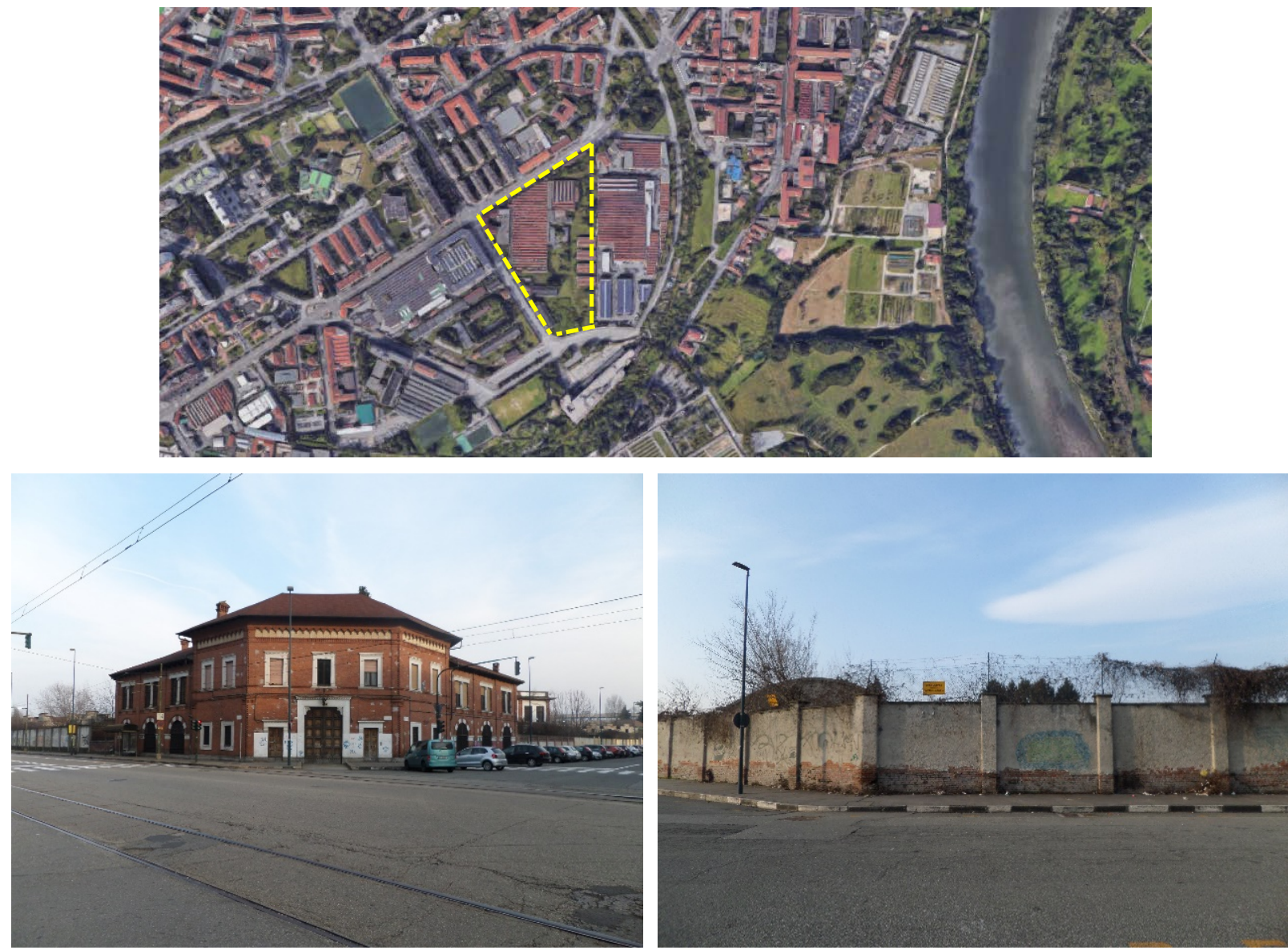

Arriba imagen de satélite y, abajo, fotografías del Almacén de Artillería y Defensa Química M.Ar.Di.Chi. (Turín), construido entre 1911 y 1928.

Fuente: Google Maps, 2018 y fotografía de Federico Camerin, 2017. 


\section{3) El desmantelamiento-vaciamiento en el marco del proceso de desposesión-apropiación de la gran propiedad militar}

Debido a su gran poder, también, a sus necesidades, el Ministerio de Defensa italiano ha ido acumulando suelo e instalaciones hasta convertirse en uno de los grandes propietarios inmobiliarios del país: según los datos de una encuesta del mismo Ministerio (Pinotti 2006), en todo el territorio nacional hay 78.300 hectáreas clasificadas como "zonas militares", lo que supone el 0,26\% de la superficie total del país. Sin embargo, el Ministerio de Defensa es un gran propietario que, habitualmente, utiliza un porcentaje bastante bajo de su patrimonio. Aun así, la enajenación ha sido siempre muy lenta, y casi siempre estaba ligada a problemas presupuestarios estatales, que son completamente diferentes con respeto a las necesidades funcionales de las Fuerzas Armadas y a aquellas que demandan de las ciudades. Como ha subrayado Insolera (1989: 667), en diversos momentos históricos se ha puesto una gran resistencia, por parte de los militares, a la enajenación de sus bienes, incluso, cuando un área ya no servía o estaba destinadas, tal vez, a otras funciones con características distintas. Frecuentemente, se ha preferido adaptarse a lo que el Ministerio de Defensa ya poseía, en lugar de enfrentarse a dos procesos, antagonistas entre ellos. Es decir, el que hace referencia a la enajenación del propio patrimonio, o la adquisición de otra propiedad. El Ministerio de Defensa siempre ha preferido mantener sus propiedades ante las imprevistas necesidades futuras, incluso, para las áreas que estaban completamente abandonadas y en estado de degradación. La lógica de su comportamiento ha sido siempre esta: si las circunstancias prevén la ocupación de nuevos espacios, la institución militar estaría preparada para equipar un área que ya está a su disposición y dentro del "sistema militar". Ciertamente, a partir de la década de 1990, en el ámbito de los procesos de enajenación de las propiedades de Defensa, se ha ido verificando la fundada preocupación por la larga (e imprevisible) duración de los procesos administrativos y urbanísticos, particularmente, relevantes cuando afecta a un aparato estatal como el militar, que puede requerir tiempos muy largos.

A partir de la caída del Muro de Berlín, en 1989, el tránsito hacia un ejército profesional, progresivamente tecnificado e inserto en una estrategia militar de alcance europeo e internacional, ha comportado unas modificaciones territoriales importantes, llevando a cabo un paulatino proceso de desmantelamiento y enajenación de grandes propiedades militares, a menudo, en estado de abandono e infrautilizadas. Por cierto, a finales de 1980, muchas de las propiedades de los ejércitos europeos no respondían a las necesidades de las Fuerzas Armadas y han quedado en zonas marginales en el nuevo marco logístico y estratégico internacional, además de estar obsoletas en comparación con un sistema de defensa moderno (o requerirían importantes recursos para adaptarse a las nuevas tecnologías, legislación y estándares). Si a ello se suman las fuertes inversiones que exigen en armamento, a partir de las últimas décadas del siglo XX y principios del siglo XXI, según Mas Hernández (2003: 239), se comprende que el abandono y la venta de muchos espacios de uso militar sea una clara tendencia en las sociedades europeas contemporáneas, siendo, en muchos casos, el propio Ministerio de Defensa quien muestra mayor interés en capitalizar las transacciones de dichos bienes con el objetivo de realizar otras inversiones.

Si bien a lo largo de las últimas tres décadas, en muchos países europeos e internacionales, se han desarrollado programas de desmantelamiento-abandono, así como sucesivas reconversiones de las instalaciones militares (para una panorámica general se remite a Bagaeen y Clark, 2016 y Ponzini y Vani, 2012; véanse, en concreto, por el caso francés Dubois-Maury, 1998 y para el caso español Brandis, Canosa, Mollá, Rodríguez y Sáez, 2005), Italia representa un caso muy particular. Efectivamente, la enajenación del patrimonio inmobiliario de la Defensa italiana (y de todas las Administraciones públicas en general), se ha constituido como un mecanismo para, en primer lugar, hacer desaparecer la titularidad pública de los derechos sobre ciertos bienes que no se consideran necesarios para su funcionamiento cotidiano. En segundo lugar, no menos importante, atendiendo a la crisis que sacude al sistema capitalista, y con el grave déficit presupuestario que sufren los Estados del mundo occidental, en Italia se ha utilizado la enajenación de estos bienes para hacer frente a la Deuda pública (Gastaldi y Camerin, 2017: 156). Otro aspecto peculiar del caso italiano es el siguiente. Según Baccichet (2015: 12), si la materialización de los asentamientos militares se ha basado en una política, tal vez, no explícitamente expresada, por el contrario, el fenómeno de desmantelamiento y abandono no ha sido el resultado de una estrategia ponderada, desarrollándose de manera completamente improvisada, por no decir anárquica.

En Italia se han producido dos acontecimientos a partir de los cuales se puede decir que, por primera vez, ha empezado el lento proceso de desmantelamiento-vaciamiento de las propiedades militares, tomándose conciencia que la expansión urbana ilimitada, y el incremento del transporte urbano, tanto de 
personas como de mercancías, habían acabado produciendo inconvenientes logísticos para los asentamientos militares que estaban insertados en tejidos urbanos densos y con mucho tráfico, convirtiéndolos en inapropiados por las exigencias de las Fuerzas Armadas. Estos momentos se refieren a los proyectos de Ley n. 148 de 6 de julio de 1972 y n. 1740 de 4 de mayo de 1989, que, sin embargo, no han sido convertidos en Ley. El primero (Senato della Repubblica, 1972) preveía el posible abandono y subasta de 351 propiedades (221 del Ejército de Tierra, 85 de la Armada y 45 del Ejército del Aire), por dos tipos de razones. Por un lado, debido a las diferentes necesidades de la organización militar moderna y, por otro, al estado vetusto en el que se encontraban muchas propiedades, las cuales ya eran innecesarias, o inadecuadas, para la función militar. El segundo, intitulado "Modernización y redistribución territorial de cuarteles e infraestructuras militares a través de un plan extraordinario de financiación decenal y subastas de inmuebles que ya son innecesarios para la Defensa" (Senato della Repubblica, 1989), se constituía como un texto más complejo con respeto al precedente, derivado de un intenso debate realizado entre finales de la década de 1970 y todo el decenio sucesivo (Aa. Vv., 1986). Ese texto introducía la posibilidad de realizar un plan de modernización de cuarteles e infraestructuras, así como la programación y la planificación de nuevos cuarteles y la reubicación de una parte de los existentes para el reequilibrio de la distribución de las Fuerzas Armadas en el territorio italiano. Incluso, se consideraba igualmente importante el objetivo de vaciar de la presencia militar los centros históricos y los ámbitos perimetrales de las grandes ciudades-capitales de regiones y provincias (por un total de más de mil infraestructuras, correspondientes a unas 35.000 hectáreas). Es decir, que el proyecto de Ley de 1989 establecía como objetivo prioritario la devolución a las ciudades de las grandes propiedades militares para el desarrollo de nuevos equipamientos y obras públicas en los respectivos contextos en los que se ubicaban y que, hasta entonces, se veían obstaculizados, o impedidos, por las limitaciones impuestas por los asentamientos militares. Sin embargo, este proyecto de Ley ha quedado sin efecto, además de no estar acompañado por un listado de las propiedades a desmantelar.

A partir de la década de 1990 se han ido promoviendo frecuentes iniciativas legislativas, en materia de gestión de las propiedades militares, para establecer, modificar o ampliar, los objetivos (predominantemente financieros) a alcanzar, así como los procedimientos para la reconversión de los activos militares, atendiendo las tareas de los actores responsables de su gestión: instrumentos y procesos han sido progresivamente cumplimentados o sustituidos por otros. Sin duda, este hecho ha multiplicado y complicado las acciones para implementar las disposiciones legislativas, configurándose como un factor problemático a la hora de analizar el nivel alcanzado por cada una de las medidas adoptadas.

En 1990, el documento de programación económica y financiera para el trienio 1991-1993 establecía, en síntesis, que el reequilibrio total de las cuentas públicas requiere una adaptación a las medidas ya adoptadas en otros países europeos, destinadas a acercar la presión fiscal al nivel de estos (Ministero dell'Economia e delle Finanze, 1990). Por eso, una de las medidas a adoptar para la consolidación de las finanzas públicas, desde entonces a finales de la década de 1990, sería la enajenación de parte de la inmensa propiedad estatal y una profunda revisión de los procedimientos de gestión del patrimonio inmobiliario del Estado, incluidas las propiedades militares. Aun así, no será hasta la Ley de Presupuestos Generales del Estado de 1997 (Parlamento italiano, 1996) cuando se inicie, oficialmente, la época del desmantelamiento-abandono y enajenación del patrimonio inmobiliario de la Defensa, con la identificación, entre 1997 y 2000, de 312 propiedades potencialmente enajenables. Desde entonces, una importante atención, por parte de algunas administraciones locales, se ha centrado en temas relacionados con el desmantelamiento-abandono y consiguientes proyectos urbanos, en el marco de procesos concretos de regeneración urbana, a partir de las propiedades militares. Por ejemplo, se destaca el empeño propiciado por la administración de la ciudad de Piacenza, desde finales de 1990 hasta hoy. De hecho, esta ciudad se caracteriza por la presencia de más de un millón de metros cuadrados de propiedades militares dentro de los confines municipales (Infussi y Pasqui, 2010).

En síntesis, los objetivos planteados en 1996 han mantenido su coherencia hasta el 2000, ya que se apoyaban sólo en tres leyes, que integraban las indicaciones iniciales: el artículo 3, párrafos 112 y 113 de la Ley n. 662/1996; el artículo 443 de la Ley n. 448/1998; el artículo 43, párrafos 7, 8, 9 y 10 de la Ley $388 / 2000$. Además, hasta aquel momento, todo el reglamento seguía conceptualmente el esquema "cambio de destinación de uso-enajenación-venta" para aquellas propiedades obsoletas para los fines de la Defensa (a través de la asignación por el período 1999-2003 de esta tarea a una empresa con participación mayoritariamente pública, la "Concessionaria Servizi Assicurativi Pubblici S.p.A.", CONSAP). Sin embargo, como se desprende de las mismas indicaciones de la Corte dei Conti (2003), 
varias cuestiones han afectado, significativamente, a la actuación del programa, determinando inevitablemente su fracaso:

- las restricciones impuestas a la enajenación de activos de interés histórico-arquitectónico-cultural, por la legislación entonces vigente;

- el considerable tiempo y presupuesto requerido para el saneamiento de ciertas propiedades destinadas a depósitos de combustibles y municiones, además de la falta de información sobre este aspecto por parte del Ministerio de Defensa;

- la posibilidad de ejercer el "derecho de prioridad" por parte de las autoridades territoriales locales, en el caso de que estuviesen interesadas en la adquisición de las propiedades militares, lo que ha implicado la necesidad de posponer las operaciones de venta, debido a la falta de recursos financieros por parte de los municipios;

- numerosas subastas se han quedado desiertas, ya que las propiedades se han considerado de baja rentabilidad en el mercado inmobiliario.

A esta fase, ha seguido una otra marcada por la inclusión de los procesos de desmantelamientovaciamiento de las propiedades militares en operaciones diferentes, las cuales han afectado a todos los bienes de propiedad del Estado, cuya gestión se ha encomendado a la Agencia del Dominio Público (incorporada a las tareas de gestión del patrimonio inmobiliario público en 1999, en el marco de la nueva organización del Ministerio de Economía y Finanzas italiano). Especificadamente, se trata de las titulaciones promovidas por el Decreto Ley n. 351 de 2001, en italiano, cartolarizzazioni; el programa Valore Paese (introducido por la Ley n. 296 de 27 de diciembre de 2006 y que comprende los proyectos Dimore, Fari y el PUVaT), y el llamado federalismo demaniale, que trata de la transferencia, a las autoridades locales, de los inmuebles de titularidad pública. Este marco fragmentado de procedimientos heterogéneos y variables ha estado acompañado por una intensa actividad legislativa en un contexto problemático-financiero para el Estado. Todo eso ha dado lugar a una paulatina superposición de roles y responsabilidades entre las diferentes administraciones institucionales, estatales y locales, por efecto de la inexperiencia en la coordinación y colaboración entre distintos niveles de gobierno, por lo que se refiere a la acción en el ámbito urbano. Para resolver los problemas de actuación, durante los primeros años del 2000, en abril de 2014 se ha introducido una task force de colaboración interinstitucional entre el Ministerio de Defensa y Agencia del Dominio Público, para fomentar nuevos procedimientos y modificar a los existentes (véanse Gastaldi y Camerin: 2017: 162-171).

Entre las nuevas iniciativas, es de destacar la venta de las instalaciones militares desmanteladas a un Fondo de Inversión Inmobiliario, el llamado "Fondo Investimenti per la Valorizzazione - Comparto Extra" (FIV) de la sociedad de gestión de activos inmobiliarios "Cassa Depositi e Prestiti sgr" (CDP). A través del Decreto del Ministerio de Economía, del 20 de diciembre de 2013, la entidad financiera privada FIV ha adquirido 40 propiedades, 33 de ellas propiedad del Estado (sobre todo, militares ya desprovistas de su uso original), y 7 de entidades territoriales, por un valor total de, aproximadamente, 490 millones de euros.

En síntesis, esta larga etapa, comenzada con el proyecto de Ley de 1972, se ha caracterizado por la creación de las condiciones básicas para la "regeneración urbana" a emprender en las ciudades. Han sido muchas las carencias, y las limitaciones, en la manera de abordar el tema del desmantelamientovaciamiento y sucesiva enajenación y reconversión de las grandes propiedades militares. A eso se añade que, hoy día, en Italia no existen investigaciones detalladas sobre los efectos y la eficacia de las operaciones puestas en marcha desde principios de 1990 que, seguramente, ayudarían a entender mejor la dimensión real de la cuestión. Las operaciones de desmantelamiento y abandono militar son un síntoma típico de la sociedad neoliberal, estrechamente vinculada con los intereses que el capital desarrolla en su intento por hacer del suelo, de todo tipo de categorías, un producto intercambiable en el mercado inmobiliario. En base al proceso de vaciamiento y abandono militar, se ha emprendido un debate político y administrativo basado en una lógica, prevalentemente financiera, de los presupuestos, y como un posible medio para la reducción de la Deuda pública. De esta manera, se han ido desplazando, a un segundo plano, otros aspectos importantes ligados a la efectiva (re)producción de la ciudad y a su gestión. El riesgo está en que la definición de nuevos espacios urbanos derivados de operaciones emprendidas sobre unas propiedades que se están convirtiendo en privadas (resultado de la enajenación de bienes de titularidad pública), pueda ser la base para llevar a cabo mensajes ideológicos que aseguren la maximización de la renta del suelo, para levantar iconos de referencia y artefactos arquitectónicos de vanguardia y espectaculares.

\section{4) La reinterpretación del antiguo cuartel militar Guido Reni en el barrio Flaminio de Roma}


El caso del cuartel Guido Reni en el barrio Flaminio de Roma constituye un ejemplo paradigmático en la manera de proceder a la reconversión de propiedades militares en grandes ciudades, a través de proyectos urbanos en el marco de procesos concretos de regeneración urbana. Esta instalación militar, llamada, también, "Fábrica de componentes eléctricos de precisión" (cuyas superficies, territoriales y brutas, alcanzan, respectivamente, 55.480 y 72.000 metros cuadrados, con un volumen total de 267.003 metros cúbicos distribuidos en 28 edificios), se encuentra en el barrio Flaminio, en una zona residencial dotada de importantes instalaciones deportivas. Esta gran propiedad militar deriva de la reconversión de una antigua y precedente área industrial para la producción de vehículos, en que tenía sede la "Società Automobili Roma", instalada allí en 1906. Después de que el Plan Regulador de Edmondo Sanjust de Teulada, de 1909, propusiese el barrio Flaminio como un ámbito especificadamente residencial, se ha decidido no modificar la destinación de uso de esa propiedad industrial para eludir su elevado coste de expropiación. Sucesivamente, en 1916, durante la Primera Guerra Mundial, debido a las necesidades bélicas de aquel momento, la antigua instalación industrial fue convertida, a través de una reestructuración, en una fábrica para la producción de armas y municiones, llamada, inicialmente, "Regia Fabbrica d'Armi". Una vez terminada la guerra, y consolidado el régimen fascista, el Plan Regulador de 1931 confirmaba y fomentaba una edificación residencial intensiva en todo el barrio Flaminio, con la previsión de convertir a viviendas, también, la instalación militar. A pesar de eso, a finales de la década de 1940, no todas las previsiones de urbanización del área de Flaminio han sido respectadas, incluida la de la reconversión de la fábrica de armas, debido a la gran extensión de la instalación militar, que ha incidido notablemente en la actuación del Plan. Por lo que respecta a la propiedad militar en sí, no se han registrado modificaciones substanciales, solamente algunas transformaciones del interior de los edificios por las exigencias de los militares. La única novedad ha sido el cambio de denominación en "Fábrica de componentes eléctricos de precisión": el sitio militar permanecerá en uso utilizo hasta finales del siglo XX, cuando se ponga fin a la presencia militar, manteniéndose, aún, como propiedad del Ministerio de Defensa.

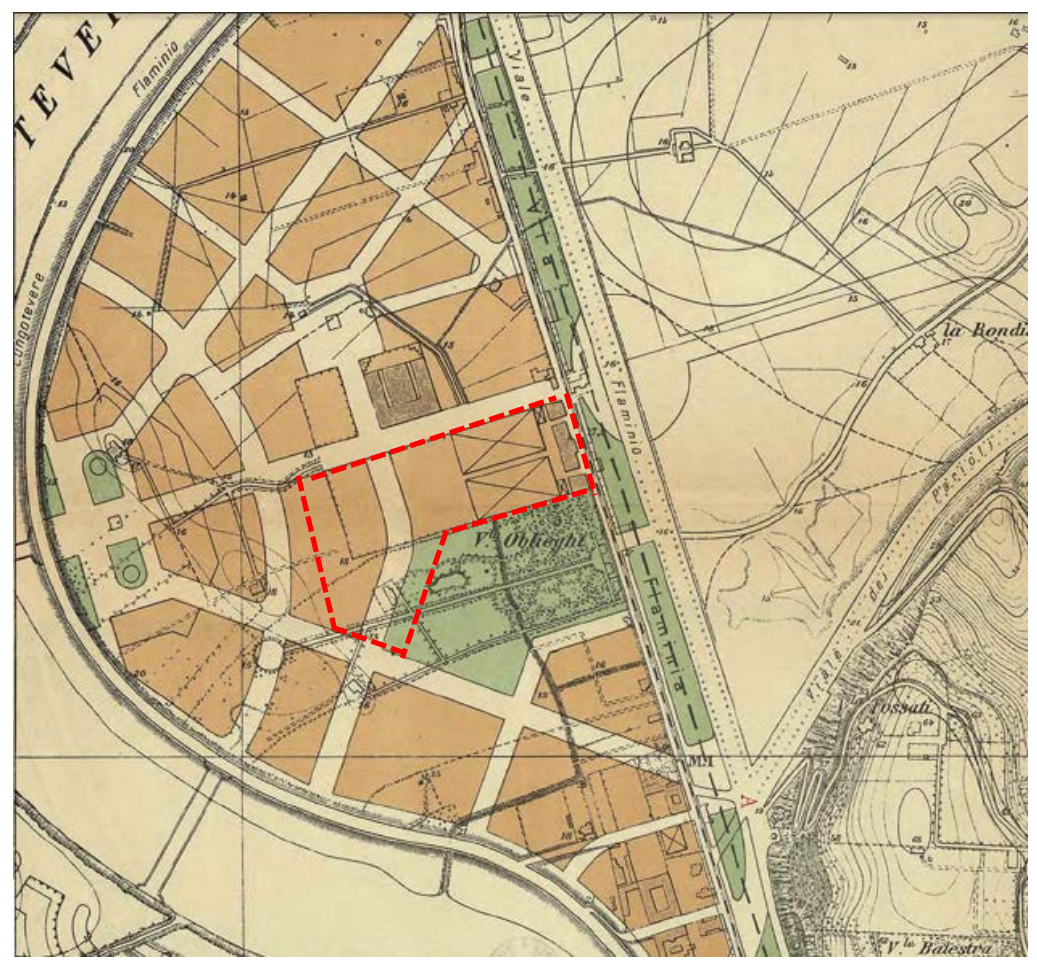

Extracto del Piano Particolareggiato del barrio Flaminio (1909). En rojo la localización de la industria de automóviles. Elaboración propia a partir del Plan Regulador General de Roma de 1909 


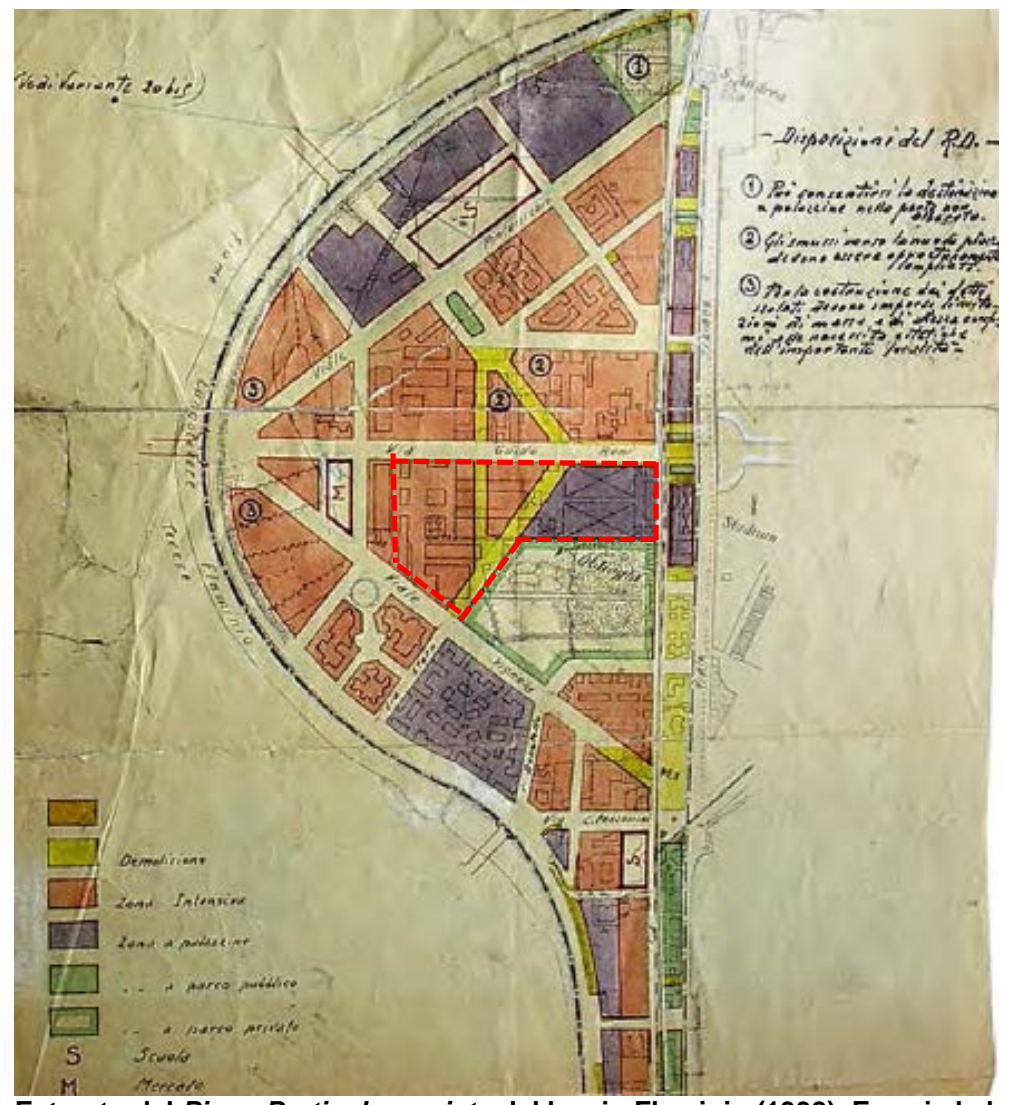

Extracto del Piano Particolareggiato del barrio Flaminio (1932). En rojo la localización de la fábrica de armas.

Elaboración propia a partir del Plan Regulador General de Roma de 1931.

A principios del nuevo siglo, con la aprobación del proyecto urbano "El Barrio de la Ciudad de la Cienciael Proyecto Urbano Flaminio" (Roma Capitale, 2005), se establece el área del cuartel Guido Reni como zona para reutilizar como equipamiento público de barrio (en italiano attrezzature di servizi pubblici di quartiere), a través de un programa de recalificación urbana. Sin embargo, después de varias vicisitudes administrativas y burocráticas (véanse Geusa, 2012 y Roma Capitale, 2014: 2-4) no será hasta 2013 cuando cambie la situación. La Agencia del Dominio Público, el Ayuntamiento de Roma y la Cassa Depositi e Prestiti, promueven la venta de la propiedad militar al Fondo FIV, acordándose el cambio de destinación de uso del antiguo cuartel a futura "Ciudad de la Ciencia", en conformidad con el proyecto urbano de 2005 (Roma Capitale, 2013) y a través de un concurso internacional para la redacción del masterplan de intervención en el área.

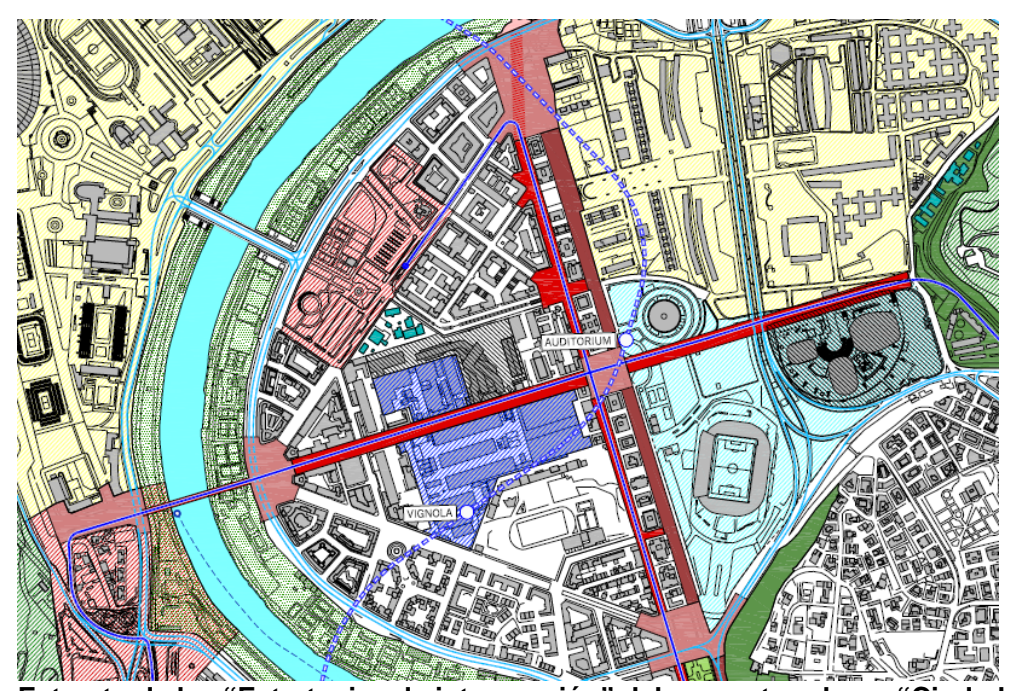

Extracto de las "Estrategias de intervención" del proyecto urbano "Ciudad de la Ciencia" (2005). El área del cuartel Guido Reni se ha clasificado como "grande infraestructura para rehabilitar" (en azul).

Fuente: proyecto urbano "Ciudad de la Ciencia" (Roma Capitale. 2005). 
El concurso, desarrollado entre 2014 y 2015, ganado por una propuesta del estudio de Paola Viganò, se ha basado en tres ejes principales: la materialización de la llamada Ciudad de la Ciencia; el impulso para la creación de un sistema de espacios públicos, con el que se vinculen equipamientos y servicios para la ciudadanía; así como, la construcción de una zona residencial privada con una cuota de alojamientos sociales. La superficie total sobre la que se actúa es de 51.000 metros cuadrados, de los que 14.000 tienen que dedicarse a espacios públicos y 10.000 a la Ciudad de la Ciencia. El resto, 27.000 metros cuadrados, constituye la cuantía del espacio residencial privado. Estos metros cuadrados, medidos en superficie de suelo dedicada a la actividad inmobiliaria residencial, se prevé que produzcan 29.000 metros cuadrados de edificabilidad para viviendas en venta, o alquiler, así como otros 6.000 metros cuadrados de edificabilidad para alojamientos sociales. A todo esto hay que añadir unos 10.000 metros cuadrados más construidos para usos turísticos y comerciales, sin contar con amplias superficies dedicadas al uso de aparcamientos. Se prevé que el coste total para la realización del masterplan sea de 350 millones de euro.

El planteamiento de la reconversión del cuartel Guido Reni se manifiesta como un paso más hacia la creación de un nuevo sector "de consumo" en la capital italiana, que pueda dar el impulso necesario a la definitiva terciarización del barrio Flaminio, lugar típicamente residencial y popular de la ciudad. A partir de la transformación de otras grande propiedades urbanas alrededor del antiguo cuartel, que han implicado la realización del Auditorium (55.000 metros cuadrados, 1994-2002) y del museo MAXII (alrededor de 30.000 metros cuadrados, 1998-2009) respectivamente, por parte de los arquitectos estrellas Renzo Piano y Zaha Hadid, el Barrio de Flaminio se está convirtiendo, paulatinamente, en un ámbito marcado por piezas arquitectónico-urbanística de naturaleza publicitaria para fomentar su prestigio de la ciudad, aunque contribuya a hacerla más desequilibrada y segregada.

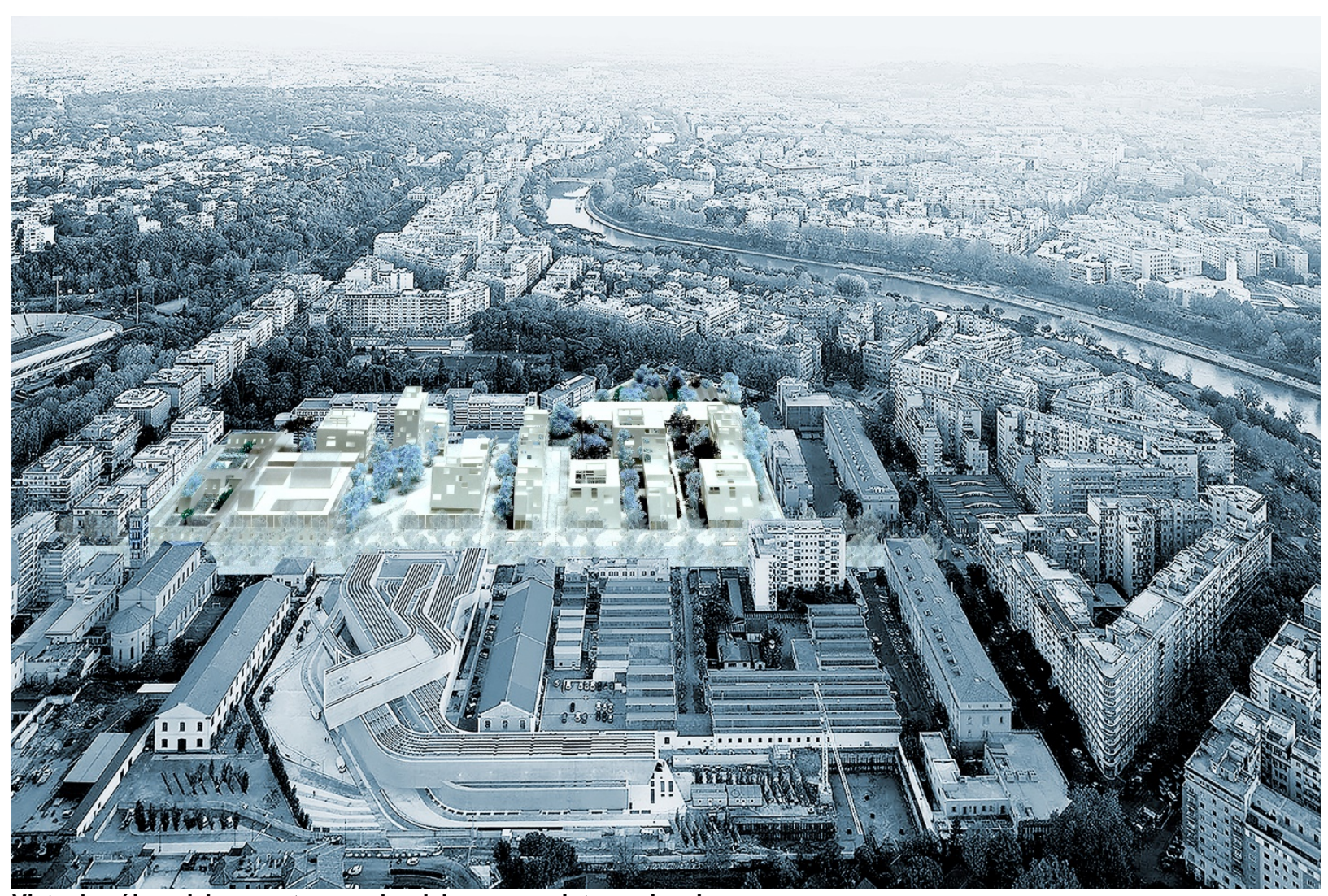

Vista de pájaro del proyecto ganador del concurso internacional.

Fuente: Studio Paola Viganò, 2015, http://www.progettoflaminio.it/press/

A partir de la década de 1980, la transformación de las ciudades italianas ha afectado a las áreas abandonadas (de tipo industrial, ferroviario y público), es decir, a las propiedades que, estratégicamente, eran los más importantes para los objetivos inherentes a cualquier proyecto urbanístico tendente a la reorganización y recualificación de ciertos sectores de la ciudad. Esto es lo que ha sucedido en distintos espacios urbanos de la ciudad, como es el caso de los Centros Históricos o "frentes marítimos", 
desencadenando procesos de regeneración que constituyeron un motor de cambio importante en la ordenación urbana y en la configuración de la ciudad. Pues, si por un lado, las ciudades han procedido a una paulatina reutilización de los vacíos postindustriales, a partir de la caída del muro de Berlín en 1989, se generaron otro tipo de "vacíos". Esta vez, procedentes de las instalaciones militares desmanteladas, que en seguida se han caracterizado, primero, por un estado de infrautilización y, segundo, por el definitivo abandono y degradación. Hoy día estos "deshechos" representan una ocasión única para devolver a la colectividad espacios que ésta misma necesita para desarrollar su vida urbana, ocasión de (re)apropiación de bienes de titularidad pública, es decir de artefactos que pertenecen de derecho a la ciudadanía. Sin embargo, a través de la actitud de los actores involucrados en los procesos de regeneración urbana (entidades estatales y locales, agentes inmobiliarios y capital financiero) y las disposiciones de los instrumentos de planificación urbana local, las operaciones emprendidas, como es el caso del cuartel Guido Reni, se están convirtiendo en un pretexto para proporcionar más espacios aptos para la mercantilización de la ciudad, además de constituir la base para su gentrificación. Como observado en el caso de estudio, esta actitud llevará a la privatización de espacios que sin embargo deberían ser devueltos a la colectividad bajo la forma de equipamientos o residencias para la población local, en un barrio que históricamente se ha caracterizado por ser un ámbito popular.

\section{Conclusiones}

Las áreas militares en abandono del siglo XXI constituyen una oportunidad para experimentar un nuevo estilo de gobierno de la ciudad para la restitución de estos bienes a los territorios de pertenencia. Las operaciones a emprender deberían ayudar a definir y explicitar la idea de ciudad que se persigue, así como el marco de los escenarios futuros, incluso, constituir un estímulo que ayude a los actores a tomar conciencia de la potencialidad y de los valores de la ciudad, ya sean los más evidentes o los que, todavía, han de ser desvelados.

Descubrir el sentido que adquiere el espacio urbano actual, identificado con las grandes propiedades militares desmanteladas y enajenadas, principalmente, en beneficio de operadores inmobiliarios privados, no sería posible sin recurrir al análisis histórico realizado a propósito de la aparición de los artefactos militares en la ciudad y su razón de ser en ámbito urbano. Se trata de un trabajo que interpreta la ciudad actual, específicamente, el caso del barrio Flaminio de Roma, remitiendo, para ello, a momentos históricos fundamentales (entre ellos, las vicisitudes de las estrategias de desmantelamiento y vaciamiento de las instalaciones militares), estableciendo una relación entre la herencia militar recibida del pasado, el planeamiento urbanístico y sus efectos en el desarrollo urbano. El antiguo compartimento militar de Guido Reni constituye una pieza clave que ha dejado su huella en la ciudad durante el siglo XX, ya sea permaneciendo, como tal, en su morfología urbana, incluso condicionando las transformaciones urbanísticas que remiten a la situación actual.

Del análisis propuesto se demuestra que, frente a las debilidades del marco de referencia, sobre el cual se está llevando a cabo el desmantelamiento-abandono militar y su reinterpretación en las grandes ciudades, los actores públicos a nivel estatal y local están elaborando unas estrategias claras para la ordenación y la gestión del territorio hacia la sociedad del consumo. De hecho, la manera en que la ciudad asume las consecuencias derivadas de las presiones directas del capital, se desemboca aumentando la apropiación de plusvalías a través de la "renta de suelo". En el caso de estudio propuesto, el capital financiero (personificado por el Fondo FIV de Cassa Depositi e Prestiti), aprovecha la exigencia del Estado por recuperar dinero, para reducir la Deuda Pública, pretendiendo valorizar el suelo de antiguas propiedades públicas con operaciones de transformación urbana costosas e intensivas. Como queda demostrado, con el barrio de Flaminio, la "regeneración urbana" a partir de la gran propiedad (en este caso, militar) está generando un desarrollo urbano basado en una especie de mosaico de representaciones temáticas aisladas. Eso está teniendo como consecuencia la desaparición de los caracteres sociales, culturales, es decir, de la propia historia del lugar.

El tratamiento de esta gran propiedad en el marco de un más amplio proceso de regeneración urbana es consecuencia de una política ya establecida anteriormente, y materializada parcialmente, pieza por pieza, a través de la realización del Auditorium y del Museo MAXII. La reconversión de los antiguos espacios militares de Guido Reni podría propulsar, de manera definitiva, la conversión de los espacios tradicionales, como aquellos del barrio de Flaminio, en ámbitos exclusivos, marcando distancias insalvables con respecto al resto de la ciudad, creando una imagen hacia el exterior, elevando los productos de consumo, tanto los perecederos como los inmobiliarios, haciéndolo de uso exclusivo de una minoría. En suma, impulsando una sociedad clasista, insostenible y ausente de cohesión social. 


\section{BIBLIOGRAFIA}

AA. VV. (1986). Conferenza nazionale sulle infrastrutture militari: atti. Roma, Ministero della Difesa.

BAGAEEN, S. y CLARK, C. (2016). Sustainable regeneration of former military sites. Londres y Nueva York: Routledge.

BETTINARIA, P. (1989). Lo stanziamento dell'esercito italiano in età liberale, En AA. VV., Esercito e città dall'Unità d'Italia agli anni Trenta. Atti del Convegno di studi Spoleto 11-14 maggio 1988. Tomo I (5-20). Roma: Ministero per i beni culturali e ambientali-Ufficio centrale per i beni archivistici.

BRANDIS, D., CANOSA, E., MOLLÁ, M., RODRÍGUEZ, I. y SÁEZ, E. (2005). La reconversión del espacio militar en Madrid: su reutilización en los últimos veinticinco años. Ciudad y Territorio. Estudios territoriales, 144, 391-415.

BRUNI N., 1937, Igiene militare, Milán: Società Editrice Libraria.

CHRISTOPHERS, B. (2017). The State and Financialization of Public Land in the United Kingdom. Antipode, 49, 1, 62-85.

CORTE DEI CONTI (2003), Alienazione di beni (art. 43 e 49 Legge Finanziaria n. 388/2000). Deliberazione 7 maggio 2003 n. 29/2003/G. Roma, Sezione centrale di controllo sulla gestione delle Amministrazioni dello Stato http://www.corteconti.it/export/sites/portalecdc/_documenti/controllo/sez_centrale_controllo_amm_stato/ 2003/delibera_29_2003_g_.pdf

DUBOIS-MAURY, J. (1998). Impacts urbains des restructurations de l'appareil militaire en France. Annales de Geographie, 599, 89-97.

GASTALDI F., CAMERIN F. (2012). Immobili pubblici e aree militari dismesse: 'occasioni' per le città italiane, fra ritardi e incertezze. Quaderni Regionali, 3, 441-460.

- (2017). La regeneración urbana en Italia a partir de la reutilización del patrimonio público inmobiliario: un catálogo de herramientas y protocolos. Ciudades, 20, 155-178.

GEUSA, M. (2012). Aree militari dismesse e trasformazioni urbane: l'esperienza di Roma. Territorio, 62, 29-33.

HARVEY, D. (2005). A Brief History of Neoliberalism. Oxford: Oxford University Press.

INFUSSI, F. y PASQUI, G. (2010). Masterplan per le aree militari di Piacenza. Primo rapporto. Milán: Politecnico di Milano, Dipartimento di Architettura e Pianificazione.

INSOLERA I. (1989), Insediamenti militari e trasformazioni urbane. En AA. VV., Esercito e città dall'Unità d'Italia agli anni Trenta. Atti del Convegno di studi Spoleto 11-14 maggio 1988. Tomo II (663-675). Roma: Ministero per i beni culturali e ambientali-Ufficio centrale per i beni archivistici.

JEAN C. (ed.) (1989), Storia delle forze armate italiane dalla ricostruzione postbellica alla ristrutturazione del 1975. Milán: Giuffrè.

MALATESTA, L., TREVISAN, G., POZZA A. y DE CASTRO C. R. (2015), Viaggio nelle basi segrete della NATO West Star e Back Yard, Varese: Pietro Macchioni Editore.

MARKUSEN, A., JUDKEN, J. (1992). Dismantling the Cold War Economy. Nueva York: Basic Books.

MAS HERNÁNDEZ, R. (2003). La presencia militar en las ciudades. Orígenes y desarrollo del espacio urbano militar en España. Madrid: Los Libros de la Catarata.

MELIS, A. (1941). Le città militari. Urbanistica. Rivista dell'Istituto Nazionale di Urbanistica, 10, 2, 15-17. 
MINISTERO DELL'ECONOMIA E DELLE FINANZE (1990). Documento di Programmazione economicofinanziaria relativo alla manovra di Finanza pubblica per gli anni 1991-1993, Roma. http://www.dt.tesoro.it/export/sites/sitodt/modules/documenti_it/analisi_progammazione/documenti_progr ammatici/DPEF_1991-1993_xpubb.1990x.pdf (consulta: 14/01/2018)

PARLAMENTO ITALIANO (1996), Legge 23 dicembre 1996, n. 662 "Misure di razionalizzazione della finanza pubblica", Gazzetta Ufficiale n. 303 del 28 de diciembre de 1996. http://www.camera.it/parlam/leggi/96662l.htm (consulta: 14/01/2018)

PINOTTI, R. (2006). Indagine conoscitiva. Roma, Ministerio de la Defensa, Commissione IV Difesa. C http://www.camera.it/_dati/leg15/lavori/stencomm/04/indag/militari/2006/1025/s000r.htm (consulta: 14/01/2018)

PONZINI, D. y VANI M. (2012). Apprendere dalle città italiane ed europee: politiche, progetti e competenze per gli immobili militari. Territorio, 62, 64-66.

ROMA CAPITALE (2005), I/ Quartiere della Città della Scienza - Il Progetto Urbano Flaminio. Roma, Dipartimento Programmazione e Attuazione Urbanistica, http://www.urbanistica.comune.roma.it/partecquart-scienza/puf.html (consulta: 14/01/2018)

ROMA CAPITALE (2013). Il Quartiere della Città della Scienza - L'area dell'ex caserma di Via Guido Reni. Roma, Dipartimento Programmazione e Urbanistica. http://www.urbanistica.comune.roma.it/partec-quart-scienza/excaserma.html (consulta: 14/01/2018)

ROMA CAPITALE (2014). Ex-“Stabilimento militare materiali elettonici e di precisione" sito en via Guido Reni. Individuazione della Zona di Recupero del patrimonio edilizio esistente e dell'ambito per la formazione del Piano di Recupero", ai sensi degli artt. 27 e 28 della L. 457/78, Roma, Assesorato alla Trasformazione Urbana, http://www.urbanistica.comune.roma.it/images/partecipazione/guido-reni/elab1relazione-08-2014.pdf (consulta: 16/01/2018)

SENATO DELLA REPUBBLICA (1972). Disegno di legge n. 148 del 6 luglio 1972: Dismissione di immobili militari ed assegnazione di fondi per il potenziamento delle Forze Armate. Roma: VI Legislatura. https://www.senato.it/service/PDF/PDFServer/DF/279315.pdf (consulta: 16/01/2018)

SENATO DELLA REPUBBLICA (1989). Disegno di legge n. 1740 del 4 maggio 1989: Ammodernamento e redistribuzione territoriale delle caserme e delle infrastrutture militari mediante un finanziamento decennale straordinario e attraverso permute ed alienazioni di immobili non più necessari alla Difesa, Roma: $X$ Legislatura. https://www.senato.it/service/PDF/PDFServer/DF/270278.pdf (consulta: $16 / 01 / 2018)$

SERVIZIO STUDI DEL SENATO (2009). Disegno di legge A.S. n. 1373 «Misure a tutela dei segni distintivi delle Forze armate e costituzione della Società "Difesa Servizi Spa". Dossier n. 100, Ufficio ricerche nel settore della politica estera e della difesa, Roma. http://www.senato.it/service/PDF/PDFServer/BGT/00736490.pdf (consulta: 15/01/2018)

TURRI, F., CAPPELLETTI, V. y ZAMPERINI E. (2008). II recupero delle caserme: tutela del patrimonio e risorsa per la collettività. Territorio, 46, 72-84.

VACIAGO, G. (2007). Gli immobili pubblici... ovvero, purché restino immobili. En MATTEI, U. et al. (coords.). Invertire la rotta: Idee per una riforma della proprietà pubblica (325-339). Bolonia: II Mulino.

WHITESIDE, H. (2017). The state's estate: devaluing and revaluing 'surplus' public land in Canada. Environment and Planning $A$. 